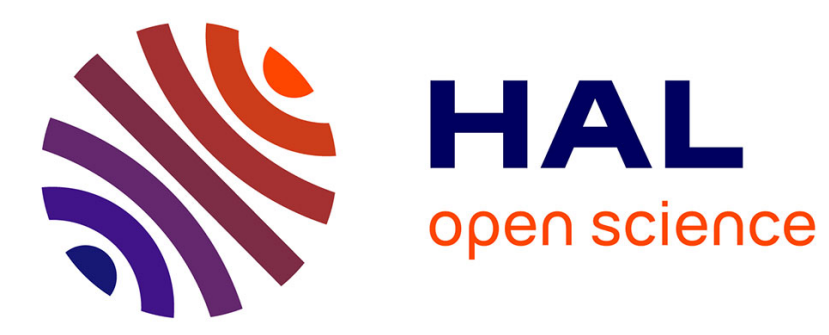

\title{
Hydrodynamic phonon transport in bulk crystalline polymers
}

Zhongwei Zhang, Yulou Ouyang, Yangyu Guo, Tsuneyoshi Nakayama, Masahiro Nomura, Sebastian Volz, Jie Chen

\section{To cite this version:}

Zhongwei Zhang, Yulou Ouyang, Yangyu Guo, Tsuneyoshi Nakayama, Masahiro Nomura, et al.. Hydrodynamic phonon transport in bulk crystalline polymers. Physical Review B, 2020, 102, 10.1103/physrevb.102.195302 . hal-03085827

\section{HAL Id: hal-03085827 https://hal.science/hal-03085827}

Submitted on 22 Dec 2020

HAL is a multi-disciplinary open access archive for the deposit and dissemination of scientific research documents, whether they are published or not. The documents may come from teaching and research institutions in France or abroad, or from public or private research centers.
L'archive ouverte pluridisciplinaire HAL, est destinée au dépôt et à la diffusion de documents scientifiques de niveau recherche, publiés ou non, émanant des établissements d'enseignement et de recherche français ou étrangers, des laboratoires publics ou privés. 


\title{
Hydrodynamic phonon transport in bulk crystalline polymers
}

\author{
Zhongwei Zhang, ${ }^{1,2,3}$ Yulou Ouyang, ${ }^{1,2}$ Yangyu Guo, ${ }^{3}$ Tsuneyoshi Nakayama $\odot,{ }^{1,2,4}$ Masahiro Nomura $\odot,{ }^{3}$ \\ Sebastian Volz, ${ }^{2,5, *}$ and Jie Chen $\circledast^{1,2, \uparrow}$ \\ ${ }^{1}$ Center for Phononics and Thermal Energy Science, School of Physics Science and Engineering, Tongji University, Shanghai 200092, \\ People's Republic of China \\ ${ }^{2}$ China-EU Joint Lab for Nanophononics, Tongji University, Shanghai 200092, People's Republic of China \\ ${ }^{3}$ Institute of Industrial Science, The University of Tokyo, Tokyo 153-8505, Japan \\ ${ }^{4}$ Hokkaido University, Sapporo 060-0826, Japan \\ ${ }^{5}$ Laboratory for Integrated Micro and Mechatronic Systems, CNRS-IIS UMI 2820, University of Tokyo, 4-6-1 Komaba, \\ Meguro-ku, Tokyo 153-8505, Japan
}

(Received 20 April 2020; revised 21 October 2020; accepted 9 November 2020; published 23 November 2020)

\begin{abstract}
Hydrodynamic phonon transport in solids exhibits unique thermal transport behaviors, such as second sound, the Poiseuille flow, and ultrahigh thermal conductivity. However, those have been limited up to the cryogenic temperature $(\sim 1 \mathrm{~K})$ for a few materials. In this work, by employing the phonon Boltzmann transport equation, we demonstrate hydrodynamic phonon transport in organic systems such as bulk crystalline polymers. Remarkable hydrodynamic phonon transport up to $50 \mathrm{~K}$ is demonstrated for both polyacene $\left(-\mathrm{C}_{4} \mathrm{H}_{2}-\right)_{n}$ and polyacetylene $\left(-\mathrm{C}_{2} \mathrm{H}_{2}-\right)_{n}$ crystals. More interestingly, a weak phonon hydrodynamic behavior takes place in crystalline polyethylene $\left(-\mathrm{C}_{2} \mathrm{H}_{4}-\right)_{n}$ in the intermediate-temperature range around $120 \mathrm{~K}$, different from the observed hydrodynamics in most systems. The spectral phonon analysis reveals that the torsional motion of the $A_{2}$ mode causes this unique hydrodynamic behavior. A modified criterion for the emergence of hydrodynamic phonon transport is proposed which agrees quantitatively with the results from thermal conductivity and phonon drifting component calculations. This study provides physical insights into the understanding of phonon hydrodynamics in organic materials and also a reliable criterion to probe the hydrodynamic phonon transport in complex systems.
\end{abstract}

DOI: 10.1103/PhysRevB.102.195302

\section{INTRODUCTION}

Thermal transport is an important issue in the fields of phonon physics and heat management [1-4]. In addition to the well-known diffusive and ballistic thermal transport regimes [1,5], thermal phonons can also propagate hydrodynamically as a collective motion when the momentumconserving phonon-phonon scattering (normal process) is dominant [6-9]. In this phonon hydrodynamic regime, many unique transport behaviors have been demonstrated so far, such as second sound [10], the Poiseuille flow [11], ultrahigh thermal conductivity $\kappa$, and its strong size effect $[12,13]$. More recently, hydrodynamic phonon transports in new systems have received extensive research attention. For instance, hydrodynamic phonon transport and second sound were reported in two-dimensional materials $[12,14]$ and graphite [15-17].

The observation of hydrodynamic transport behavior is, however, limited to only a few inorganic materials demonstrated at very low temperatures [7,17-20]. For example, phonon hydrodynamics and second sound are experimentally observed in bismuth $[7,10]$ and solid helium [20] below $4 \mathrm{~K}$. More recently, studies have revealed that the existence of the bending phonon modes is the origin of hydrodynamic phonon transport observed in two-dimensional systems $[12,14]$ and

\footnotetext{
*volz@iis.u-tokyo.ac.jp

$\dagger$ jie@tongji.edu.cn
}

graphite [16]. In this regard, organic materials, such as crystalline polymers which have intrinsically bending phonon modes, would be a promising platform to explore phonon hydrodynamics. Moreover, the criterion to determine the emergence of phonon hydrodynamics is still an open question. In the 1960s, Guyer and Krumhansl [6] proposed a criterion to predict different phonon transport regimes by directly comparing various scattering rates. Compared to recently reported criteria, such as heat wave propagation length [7] and dimensionless $\kappa$ [16], Guyer's criterion is still the most widely used one. However, its validity in complex materials or at relatively high temperatures deserves further examination.

In this work, we report hydrodynamic phonon transport in organic systems and, more specifically, in bulk crystalline polymers. Three types of bulk crystalline polymers are studied, including polyethylene $\left(-\mathrm{C}_{2} \mathrm{H}_{4}-\right)_{n}$, polyacene $\left(-\mathrm{C}_{4} \mathrm{H}_{2}-\right)_{n}$, and polyacetylene $\left(-\mathrm{C}_{2} \mathrm{H}_{2}-\right)_{n}$ (see Fig. 1). Unlike the emergence of hydrodynamic phonon transport at low temperatures observed in polyacene (PA-I) and polyacetylene (PA-II), we find that hydrodynamic phonon transport in polyethylene (PE) unusually appears in the intermediate-temperature range, which cannot be captured by Guyer's criterion. We further propose a modified criterion based on the mode-dependent $\kappa$ that can precisely predict the hydrodynamic phonon transport regime in all three types of crystalline polymers. Our work provides physical insights into the deeper understanding of hydrodynamic phonon transport in polymer systems. 


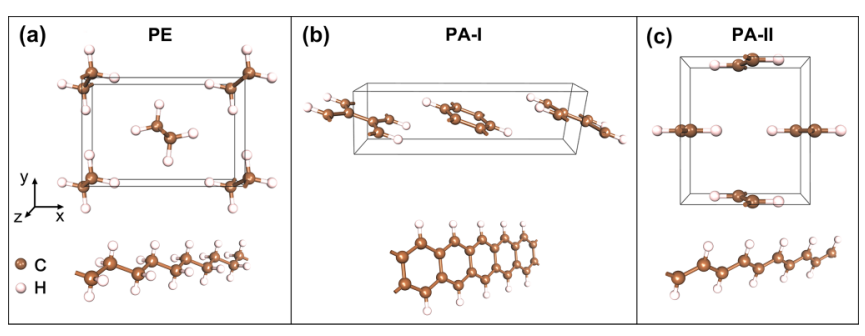

FIG. 1. The unit cell structures of crystalline polymers for (a) polyethylene $\left(-\mathrm{C}_{2} \mathrm{H}_{4}-\right)_{n}(\mathrm{PE})$, (b) polyacene $\left(-\mathrm{C}_{4} \mathrm{H}_{2}-\right)_{n}$ (PA-I), and (c) polyacetylene $\left(-\mathrm{C}_{2} \mathrm{H}_{2}-\right)_{n}$ (PA-II). The top panel is the bulk crystalline polymer, and the bottom panel is the single-chain crystalline polymer.

\section{CRYSTALLINE POLYMERS AND METHODOLOGY}

The bulk crystalline polymers are composed of wellaligned polymer chains [21,22] and inherently possess strong bending acoustic (ZA) phonon modes due to the flexible nature of polymers [23,24]. Because of the weak van der Waals (vdW) coupling between polymer chains, bulk crystalline polymers well persist the quadraticlike ZA phonon modes, which was reported in previous studies on PE [21,25]. The atomic structures of the individual polymer chain in bulk crystalline polymers are shown in Fig. 1, and the unit cell for three types of crystalline polymers is shown in the top panel of Fig. 1. More detailed information about the atomic structures can be found in the Materials Project database [26].

By using the iterative solution of the Boltzmann transport equation (BTE), the lattice thermal conductivity $\kappa_{I}$ of crystalline polymers along the axial $(z)$ direction of the polymer chains can be obtained as [27-29]

$$
\kappa_{I}=\frac{1}{k_{B} T^{2} \Omega N} \sum_{\lambda} n_{0}\left(n_{0}+1\right)\left(\hbar \omega_{\lambda}\right)^{2} v_{\lambda} F_{\lambda},
$$

where $k_{B}, T, \Omega$, and $N$ refer to the Boltzmann constant, the temperature, the volume of the unit cell, and the number of wave vector points in the first Brillouin zone, respectively. The summation goes over each phonon mode $\lambda$ that is uniquely defined by both wave vector and phonon branch. $n_{0}$ denotes the equilibrium Bose-Einstein distribution function, $\hbar$ and $\omega_{\lambda}$ are, respectively, the reduced Planck constant and the phonon frequency, and $v_{\lambda}$ is the phonon group velocity along the $z$ direction. During the iterative process, the last term, $F_{\lambda}$, in Eq. (1) can be expressed as [27,28,30] $F_{\lambda}=\tau_{\lambda}^{0}\left(v_{\lambda}+\Delta_{\lambda}\right)$, where $\tau_{\lambda}^{0}$ is the phonon lifetime of the mode $\lambda$ in the relaxation time approximation (RTA) as obtained from perturbation theory and $\Delta_{\lambda}$ is a correction term [27,29]. The phonon scattering process and the initial $\tau_{\lambda}^{0}$ are calculated based on Fermi's golden rule with the force constant obtained from the adaptive intermolecular reactive empirical bond order (AIREBO) potential [31], which has been widely used in the study of thermal transport in polymers. In the AIREBO potential, the long-range vdW interaction between polymer chains is included. The boundary scattering is not considered in the calculations of thermal conductivity since we focus on the intrinsic thermal conductivity of bulk samples. Both thermal conductivity calculations based on RTA $\left(\kappa_{R}\right)$ and the iterative solution $\left(\kappa_{I}\right)$ are performed by using the ShengBTE
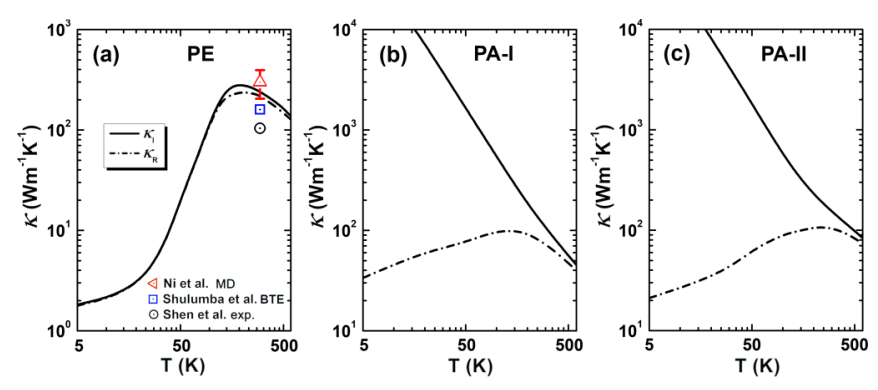

FIG. 2. Thermal conductivities of crystalline polymers from the iterative solution $\left(\kappa_{I}\right)$ and relaxation time approximation $\left(\kappa_{R}\right)$ of the Boltzmann transport equation for (a) PE, (b) PA-I, and (c) PA-II. The symbols in (a) denote the literature results from Refs. [21,32,33].

package [29]. The convergence of the integration grid along three directions in the calculation of $\kappa$ has been well tested for all systems at various temperatures. The convergent mesh is chosen to be $4 \times 6 \times 60(>75 \mathrm{~K})$ and $4 \times 6 \times 70(\leqslant 75 \mathrm{~K})$ for PE, $4 \times 7 \times 60(>75 \mathrm{~K})$ and $4 \times 7 \times 70(\leqslant 75 \mathrm{~K})$ for PA-I, and $5 \times 5 \times 60(>75 \mathrm{~K})$ and $5 \times 5 \times 70(\leqslant 75 \mathrm{~K})$ for PA-II, respectively.

\section{THERMAL CONDUCTIVITY}

The calculated thermal conductivities for three types of crystalline polymers are shown in Fig. 2. Compared with the low thermal conductivity values in amorphous polymer systems [23,24,34], which are typically on the order of 0.1 $\mathrm{W} \mathrm{m}{ }^{-1} \mathrm{~K}^{-1}$ at room temperature, the crystalline polymers exhibit much higher $\kappa$ as predicted by BTE calculations. For instance, the room temperature thermal conductivity for PE calculated from iterative solution is $216 \mathrm{~W} \mathrm{~m}^{-1} \mathrm{~K}^{-1}$, in good agreement with literature results from the molecular dynamics simulation [32], the BTE calculation [21], and experimental measurement [33]. Our results also show $\kappa$ perpendicular to the chain direction, the $x$ or $y$ direction, is about $0.22 \%$ of the value along the $z$ direction due to the weak vdW interaction, which is neglectable in this work. Moreover, the two schemes of BTE calculations are notably different in certain temperature ranges for all three types of crystalline polymers. The RTA scheme substantially underestimates the thermal conductivity compared to the iterative scheme, especially in PA-I and PA-II. A similar discrepancy in thermal conductivity has also been reported in two-dimensional materials [12] and bismuth [7]. This is because RTA incorrectly treats the normal process as a thermally resistive process that contributes directly to thermal resistance, similar to the umklapp process. However, the normal process only redistributes phonons in the reciprocal space and does not contribute directly to thermal resistance. For normal processes, total momentum is conserved $\left(\mathbf{q}_{\lambda}+\mathbf{q}_{\lambda^{\prime}}-\mathbf{q}_{\lambda^{\prime \prime}}=\mathbf{0}\right)$, which means all three phonon wave vectors belong to the first Brillouin zone, whereas in umklapp processes momentum is not conserved $\left(\mathbf{q}_{\lambda}+\mathbf{q}_{\lambda^{\prime}}-\mathbf{q}_{\lambda^{\prime \prime}}=\mathbf{G}\right)$ and the summation corresponds to a nonzero reciprocal lattice vector $(\mathbf{G})$. Here, $\mathbf{q}_{\lambda, \lambda^{\prime}, \lambda^{\prime \prime}}$ are the wave vectors of three phonon modes, $\lambda, \lambda^{\prime}$, and $\lambda^{\prime \prime}$, respectively, which participate in the three-phonon scattering processes. As a result of energy and momentum conservation, the normal processes do not directly dissipate heat, and umklapp processes act as the source of 

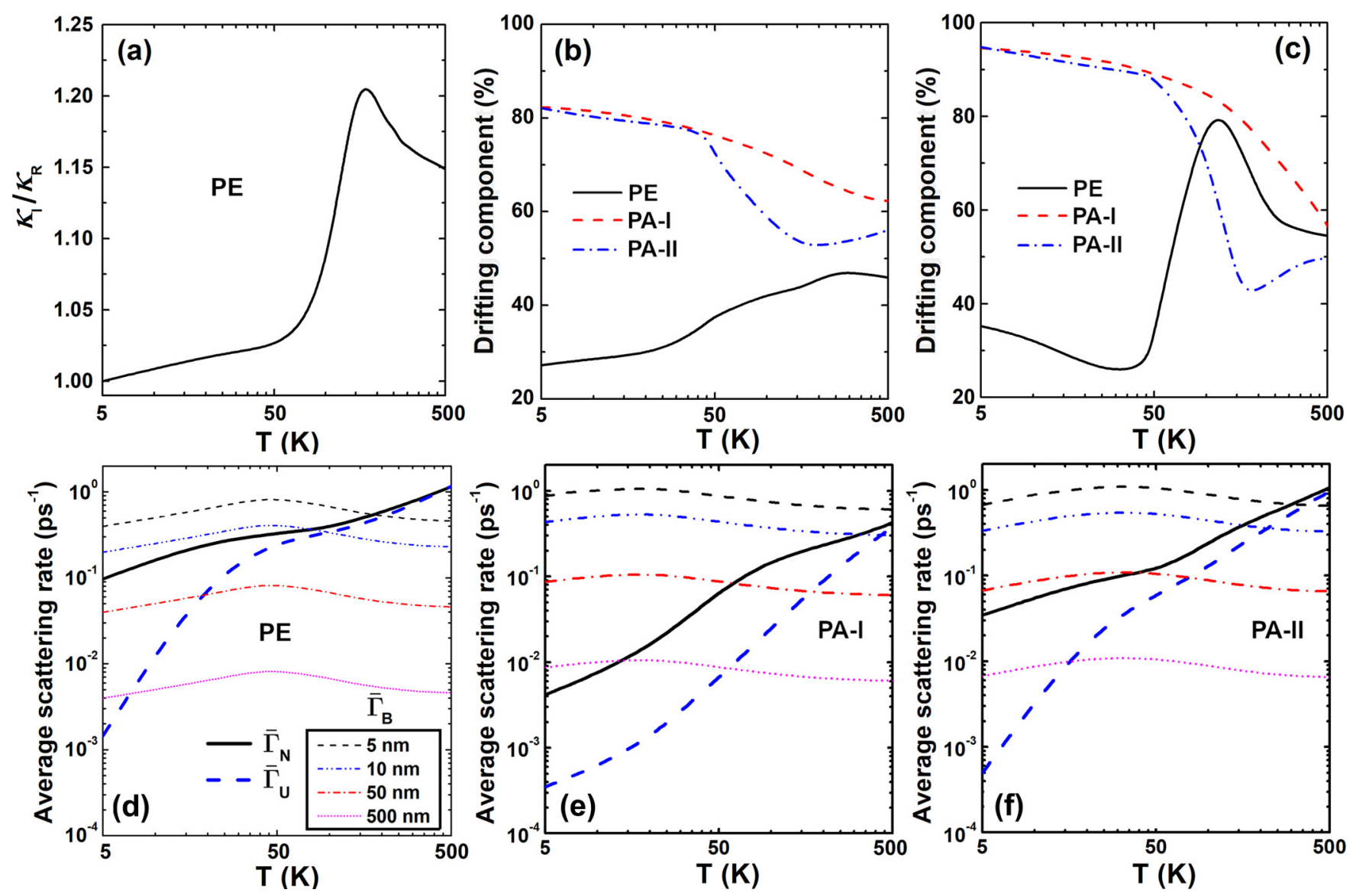

FIG. 3. (a) The ratio of $\kappa_{I} / \kappa_{R}$ in PE. (b) and (c) The averaged drifting component in crystalline polymers calculated based on Eqs. (6) and (7), respectively. The averaged scattering rates of the normal process, umklapp process, and boundary scattering calculated based on Eq. (3) for (d) PE, (e) PA-I, and (f) PA-II, respectively.

intrinsic dissipation. The equal treatment of normal and umklapp scattering in RTA calculations would overestimate the phonon-phonon scattering rate and thus cause the underestimation of $\kappa$ when normal scattering is significantly larger than the umklapp scattering.

When the umklapp process is comparable to the normal process in phonon scatterings, which is typically the case at high temperatures [35], $\kappa_{R}$ overlaps with $\kappa_{I}$ (Fig. 2). At low temperatures, however, the RTA scheme is not applicable in the study of hydrodynamic phonon transport as the normal process is dominant [36]. Interestingly, although $\kappa_{R}$ is not reliable for hydrodynamic phonon transport, its deviation from $\kappa_{I}$ indirectly reflects the importance of the normal process, which is a signature of hydrodynamic phonon transport. In other words, the difference between $\kappa_{R}$ and $\kappa_{I}$ could serve as an indicator for the emergence of hydrodynamic phonon transport. Therefore, the ratio of $\kappa_{I}$ to $\kappa_{R}$ for PE displayed in Fig. 3(a) indicates that hydrodynamic phonon transport takes place in the intermediate temperature range at $\sim 120 \mathrm{~K}$, while the large discrepancies between $\kappa_{I}$ and $\kappa_{R}$ shown in Figs. 2(b) and 2(c) suggest that hydrodynamic phonon transport occurs in both PA-I and PA-II at much lower temperatures. However, the difference between $\kappa_{I}$ and $\kappa_{R}$ is just a signature of phonon hydrodynamics. To further identify various thermal transport regimes, Guyer's criterion should be applied (see Sec. IV).
We then discuss the temperature dependence of thermal conductivity observed in Fig. 2. The temperaturedependent thermal conductivity in crystals is a consequence of the competing relationship between phonon excitation and phonon-phonon scattering. When the temperature decreases, high-frequency phonons are less excited according to the Bose-Einstein distribution, leading to the reduction of heat capacity and thus the reduction of thermal conductivity. At the same time, the anharmonic phonon-phonon scatterings are suppressed, leading to the increase of phonon lifetimes and thus the increase of thermal conductivity. As we shall demonstrate in Sec. IV, the low-frequency phonons contribute dominantly to thermal conductivity in both PA-I and PA-II due to the large group velocity [see Figs. 4(b) and 4(c)], which still can be excited at low temperatures. Therefore, the suppressed phonon scattering is the dominant competing effect in both PA-I and PA-II, leading to the increase of $\kappa_{I}$ in Figs. 2(b) and 2(c) with the decreasing temperature. However, for the case of PE, the low-frequency phonons are mixed with flat bands exhibiting low group velocity and a stronger phonon-phonon scattering rate, which causes the dominant contribution phonon to thermal conductivity shifts to high frequency (see discussion in Sec. IV). The high-frequency phonons are less excited with decreasing temperature, leading to the decreasing $\kappa_{I}$ in PE with decreasing temperature shown in Fig. 2(a). 


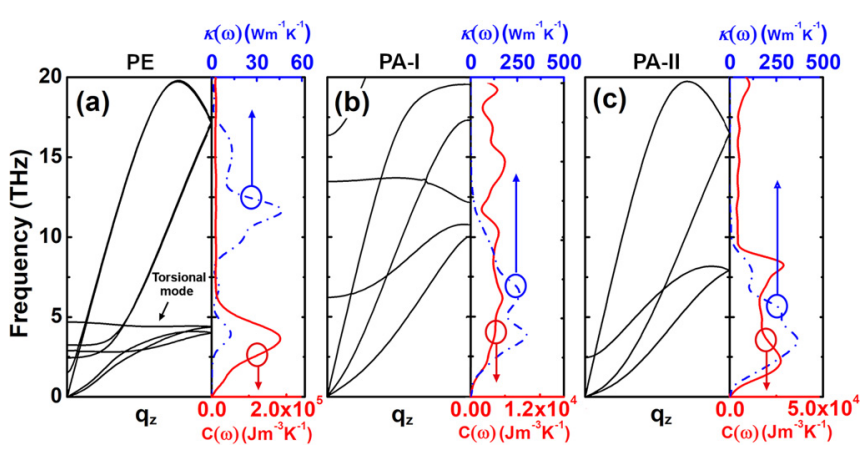

FIG. 4. Phonon dispersion (left panel) and mode-contributed specific heat $C(\omega)$ and thermal conductivity $\kappa(\omega)$ (right panel) for (a) PE at $120 \mathrm{~K}$, (b) PA-I at $50 \mathrm{~K}$, and (c) PA-II at $50 \mathrm{~K}$. The arrow in (a) indicates the torsional motion $A_{2}$ optic mode. Only the dominated phonon modes below $20 \mathrm{THz}$ are displayed.

On the other hand, the normal process, which becomes significant at low temperature, is incorrectly treated as the thermal resistive process in the RTA calculations, leading to an overestimated phonon scattering rate at low temperatures. Surprisingly, the RTA solution predicts a decreasing trend in thermal conductivity at low temperatures in both PA-I and PA-II, which is in direct contradiction to the increasing trend predicted by the iterative solution. It should be pointed out that the decreasing trend with the RTA solution at low temperatures found in our study is different from the decreasing trend induced by the boundary scattering reported in previous studies [37,38], as we consider bulk crystalline polymers and no boundary scattering term is included in our calculations. To examine the origin of such discrepancy between RTA and iterative solutions, we take PA-I as an example and compare the spectral phonon properties at two different temperatures (150 and $40 \mathrm{~K})$.

From the kinetic theory, thermal conductivity can be written as $\kappa=\sum_{\lambda} C_{\lambda} v_{\lambda} l_{\lambda}$, where $C_{\lambda}$ and $l_{\lambda}$ denote the heat capacity and mean free path for the phonon mode $\lambda$. The phonon mean free path distribution is related to the phononphonon scattering strength, which is different for RTA and iterative solutions, while the variation of heat capacity results from the temperature-dependent phonon population, which is the same for these two solutions. When the temperature decreases, two effects take place: (i) the reduced phonon-phonon scattering that leads to the increase of $l_{\lambda}$ and (ii) the decrease of phonon population that causes the decrease of $C_{\lambda}$. The final temperature dependence of the thermal conductivity is a result of these two competing effects.

Figure 5(a) shows that the values of $l_{40 K} / l_{150 K}$ for various phonons are all greater than unity, indicating that the phonon mean free path rapidly increases with decreasing temperature due to the weakened phonon-phonon scattering. However, compared to the significant increase of the phonon mean free path in the iterative solution, the enhancement of the phonon mean free path in the RTA solution is limited, exhibiting a difference of at least two orders of magnitude between the iterative and RTA solutions in the ratio of $l_{40 K} / l_{150 K}$. Such a difference should originate from the overestimated phonon-phonon scattering in the RTA solution, in which the ultrastrong normal process is not correctly treated.
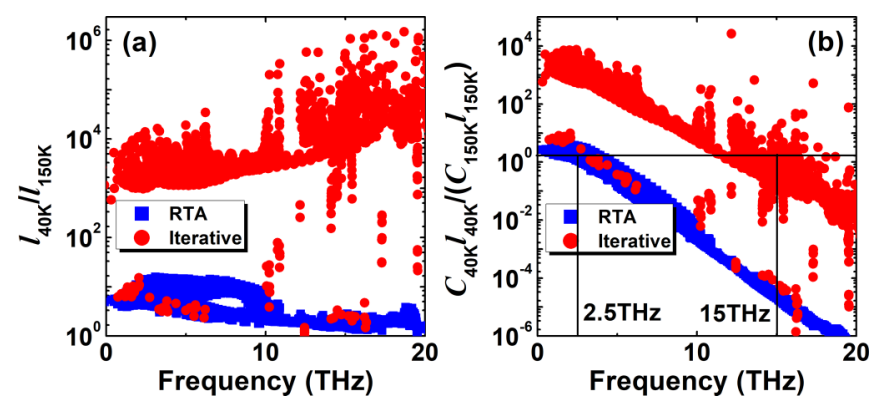

FIG. 5. Comparison of spectral phonon properties at different temperatures for PA-I. (a) The ratio of the phonon mean free path $l$ at 40 and $150 \mathrm{~K}$. (b) The ratio of heat capacity weighted phonon mean free path $\mathrm{Cl}$ at 40 and $150 \mathrm{~K}$. The calculations are carried out with RTA and the iterative solution of BTE, respectively. The solid lines in (b) are plotted to guide the eye for the ratio of unity.

The heat capacity weighted phonon mean free path, i.e., $\mathrm{Cl}$, is further shown in Fig. 5(b). The values of the ratio $C_{40 K} l_{40 K} /\left(C_{150 K} l_{150 K}\right)$ for the RTA solution are greater than unity when the frequency is less than $2.5 \mathrm{THz}$. Above $2.5 \mathrm{THz}$, the ratio of $C_{40 K} l_{40 K} /\left(C_{150 K} l_{150 K}\right)$ for the RTA solution is less than unity and shows a rapidly decreasing tendency, indicating the variation of phonon properties with temperature is dominated by the reduction of phonon population in this frequency range. The final thermal conductivity value depends on the relative contributions from the low-frequency phonons (below $2.5 \mathrm{THz}$, which have enhanced $\mathrm{Cl}$ ) and the high-frequency phonons (above $2.5 \mathrm{THz}$, which have reduced $\mathrm{Cl}$ ). The cumulative thermal conductivity in Fig. 6 further shows that phonons with frequency below $2.5 \mathrm{THz}$ contribute only around $10 \%$ to the total thermal conductivity in the RTA solution. Therefore, the phonons above $2.5 \mathrm{THz}$, which have reduced $\mathrm{Cl}$ with decreasing temperature, make the dominant contribution to thermal conductivity at low temperature for the RTA solution, which eventually leads to the decreasing trend of thermal conductivity for the RTA solution with decreasing temperature. This abnormal temperature dependence

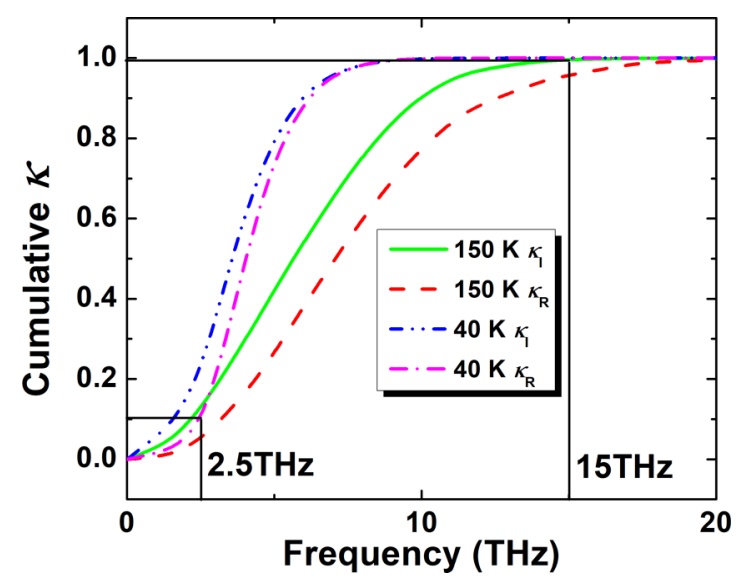

FIG. 6. The normalized cumulative thermal conductivity of PA-I computed by iterative $\left(\kappa_{I}\right)$ and RTA $\left(\kappa_{R}\right)$ solutions at 150 and $40 \mathrm{~K}$. The vertical and horizontal lines pinpoint two frequencies $(2.5$ and $15 \mathrm{THz}$ ) for comparison at $40 \mathrm{~K}$. 
is completely opposite to the increasing trend of thermal conductivity with decreasing temperature observed in most materials at low temperature where the hydrodynamic phonon transport behavior is absent, such as in bulk diamond [28] and silicon [36].

On the contrary, with the iterative solution, the threshold of unity for the ratio of $C_{40 K} l_{40 K} /\left(C_{150 K} l_{150 K}\right)$ is around $15 \mathrm{THz}$ [Fig. 5(b)], while phonons below $15 \mathrm{THz}$ make the dominant contribution to the total thermal conductivity with the iterative solution (Fig. 6), leading to the increasing trend of thermal conductivity for the iterative solution with decreasing temperature. Here, we emphasize that compared to the iterative solution, the incorrect treatment of the normal process in the RTA solution not only leads to the underestimation of the thermal conductivity but also can produce unreliable temperature dependence of the thermal conductivity at low temperature when the hydrodynamic phonon transport takes place (e.g., the normal process is dominant). Meanwhile, the observed temperature-dependent $\kappa_{R}$ is different from predictions by the Callaway model in which normal and umklapp processes are separately treated [39]. Our finding is consistent with the temperature dependence of thermal conductivity reported by Ding et al. in three-dimensional graphite with hydrodynamic phonon transport [16].

\section{CRITERION FOR HYDRODYNAMIC PHONON TRANSPORT}

To precisely determine the emergence of hydrodynamic phonon transport, one should look into the specific phonon scattering process in the reciprocal space. Several criteria $[6,7,16]$ have been applied to identify different transport regimes. Among them, the criterion proposed by Guyer and Krumhansl [6] in the 1960s is still the most widely used and determines the phonon transport regimes by comparing the averaged scattering rates. Although phonons with different frequencies may have different transport characteristics in the same system, the overall transport behavior of the system is determined by the full contribution of all phonons. Therefore, the approach of ensemble averaging among various phonons is adopted to determine the macroscopic transport behavior [12]. For Poiseuille flow, Guyer's criterion for hydrodynamic phonon transport reads [6]

$$
\bar{\Gamma}_{N} \gg \bar{\Gamma}_{B} \gg \bar{\Gamma}_{U},
$$

in which $\bar{\Gamma}_{N}, \bar{\Gamma}_{U}$, and $\bar{\Gamma}_{B}$ are the averaged normal, umklapp, and boundary scattering rates, respectively. The boundary scattering rate is defined by Casimir's theory [40] as $\Gamma_{B}=$ $2\left|v_{\perp}\right| / d$, where $v_{\perp}$ denotes the phonon group velocity in the transverse direction perpendicular to the axial direction of the polymer chain and $d$ is the sample width in the transverse direction. To obtain the average value, the scattering rate for each scattering process $s$ is usually ensemble averaged over frequencies $[6,12,14,16]$ with the following thermodynamic approach:

$$
\bar{\Gamma}_{s}=\frac{\sum C(\omega) \Gamma_{s}(\omega)}{\sum C(\omega)},
$$

where $C(\omega)=n_{0}(\omega)\left[n_{0}(\omega)+1\right](\hbar \omega)^{2} /\left(k_{b} T^{2}\right)$ is the mode specific heat. In Guyer's original study, the Debye specific heat was used in this averaging formula. However, considering the quantum phonon population from the Bose-Einstein distribution is applied in the BTE calculations and phonon hydrodynamics happen at low temperatures, Einstein's specific heat expression is adopted in this work as reported in other BTE studies [7,12,16].

Following Guyer's criterion, the averaged scattering rates for PE are compared in Fig. 3(d). Obviously, the averaged boundary scattering rate that satisfies Eq. (2) falls in the width range from $\sim 50$ to $500 \mathrm{~nm}$, and hydrodynamic phonon transport occurs in the low-temperature range (below $50 \mathrm{~K}$ ). This prediction directly contradicts the thermal conductivity ratio shown in Fig. 3(a), which suggests that a weak hydrodynamic phonon transport predominantly takes place in the intermediate-temperature range around $120 \mathrm{~K}$. On the other hand, Guyer's criterion predicts the emergence of hydrodynamic phonon transport at low temperature for both PA-I and PA-II [see Figs. 3(e) and 3(f)], which agrees qualitatively well with the direct observation of the discrepancy in $\kappa$ shown in Figs. 2(b) and 2(c). These results reveal that Guyer's criterion [Eqs. (2) and (3)] might not hold in complex systems such as PE.

To investigate the origin for the failure of Guyer's criterion in $\mathrm{PE}$, we compare the phonon properties for three types of crystalline polymers at the temperatures where the discrepancy between $\kappa_{I}$ and $\kappa_{R}$ appears (see Fig. 4). Due to the large population of bending acoustic phonons, the spectral thermal conductivity $\kappa(\omega)$, here, $\kappa_{I}=\Sigma \kappa(\omega)$, in both PA-I and PA-II is dominantly contributed by the bending acoustic phonons below $10 \mathrm{THz}$, similar to the case for graphene [41]. Consistently, the dominant contribution of $C(\omega)$ in both PA-I and PA-II is also from low-frequency phonons. Therefore, both Guyer's criterion and $\kappa$ discrepancy can qualitatively provide the same prediction for the emergence of hydrodynamic phonon transport at low temperature in both PA-I and PA-II.

In sharp contrast, the highly structural anisotropy and cylinderlike molecular segment in PE result in a torsional motion [42,43], as shown in Fig. 7(a), which gives rise to the torsional $A_{2}$ mode observed in the phonon dispersion [Fig. 4(a)]. However, such torsional motion does not exist in the other two types of bulk crystalline polymers for the entire frequency range due to their planelike molecular segments. Interestingly, the flat band of the $A_{2}$ mode in PE provides pronounced scattering channels for phonon-phonon scattering. In addition, this $A_{2}$ mode can easily hybridize with TA and ZA modes, as shown in Fig. 7(b). This hybridization can notably enhance phonon anharmonicity for the hybridized phonon modes $[44,45]$. These two factors should have significant effects on the detailed phonon-phonon scattering.

To further explore the impact of the torsional mode on the hydrodynamic transport in PE, we examine in Figs. 7(c) and 7(d) the phonon scattering rate for both normal and umklapp processes in PE when the hydrodynamic transport occurs at $120 \mathrm{~K}$. Due to the low-frequency and flat-band features of the torsional mode in $\mathrm{PE}$, the participation of the torsional mode provides a large number of scattering channels for the low-frequency acoustic phonons. Moreover, the hybridization between the torsional band and acoustic bands [see Fig. 7(b)] results in the strong anharmonicity in the low-frequency 

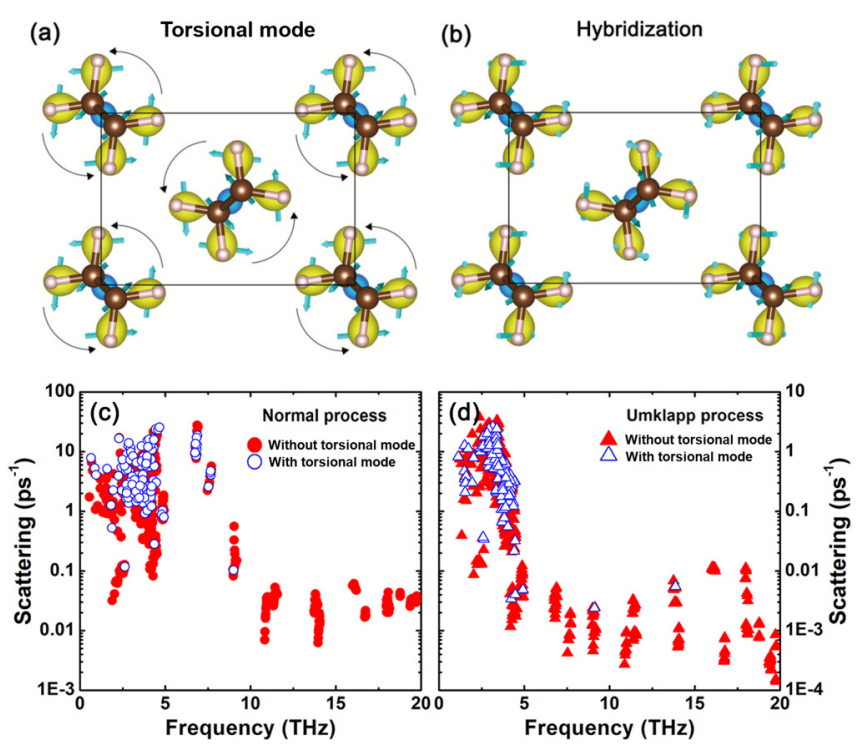

FIG. 7. Unique transport phenomenon induced by the torsional mode in PE. Schematic figure of lattice vibration for (a) the torsional mode at the zone center and (b) phonon hybridization at the zone boundary. The arrows indicate the vibration direction of the phonon mode. (c) and (d) The three-phonon scattering rates in PE at $120 \mathrm{~K}$ for normal and umklapp processes, respectively. The solid and open symbols denote the three-phonon scattering process without and with the participation of torsional mode, respectively.

region, regardless of the participation of the torsional mode in the scattering process. Consequently, Figs. 7(c) and 7(d) reveal that the torsional mode has a dominant impact on the low-frequency phonons below $5 \mathrm{THz}$, leading to the large scattering rate in this low-frequency regime. This is the origin of the suppressed contribution from low-frequency phonons to the thermal conductivity and the dominant contribution of high-frequency phonons in PE as observed in Fig. 4(a). In addition, Figs. 7(c) and 7(d) also reveal that the participation of the torsional mode (open symbols) in the three-phonon scattering process results in a notably higher scattering rate compared to the case without torsional mode (solid symbols) in the low-frequency regime below $5 \mathrm{THz}$ for both normal and umklapp processes, presumably due to the enhanced number of scattering channels with the participation of the torsional mode mentioned above. Another important feature is the phonon scattering rate for the normal process [Fig. 7(c)] is much higher than that for the umklapp process [Fig. 7(d)], leading to the hydrodynamic transport in PE observed at $120 \mathrm{~K}$.

On the other hand, the hybridization significantly lowers the acoustic modes to the low-frequency region below $5 \mathrm{THz}$, where the dominant peak in $C(\omega)$ is located. This effect causes not only a reduced group velocity of acoustic phonons but also the enhanced overall phonon-phonon scattering rates of low-frequency phonons [see Figs. 7(c) and 7(d)], leading to the suppressed thermal transport for low-frequency phonons. However, the high-frequency phonons above $5 \mathrm{THz}$ in $\mathrm{PE}$, which still have large group velocity and a relatively low scattering rate, are less affected. As a result, the dominant peak in $\kappa(\omega)$ shifts to a higher frequency of $\sim 11.3 \mathrm{THz}$, causing a complete separation of the dominant spectrum peak between $\kappa(\omega)$ and $C(\omega)$. Therefore, the breakdown of Guyer's criterion in $\mathrm{PE}$ is caused by the frequency separation between the dominant peak spectrum of $\kappa(\omega)$ and $C(\omega)$, so that the thermodynamic average in Eq. (3) based on $C(\omega)$ cannot reliably capture the thermal transport behavior in PE.

To overcome such inconsistency, we propose a modified criterion using $\kappa(\omega)$ obtained from the iterative solution as the weighting function in the ensemble average as

$$
\bar{\Gamma}_{s}=\frac{\sum \kappa(\omega) \Gamma_{s}(\omega)}{\sum \kappa(\omega)} .
$$

With this modification, the recalculated averaged scattering rates indeed confirm that our modified criterion can correctly capture the unusual feature in PE; that is, $\bar{\Gamma}_{N} \gg \bar{\Gamma}_{U}$ is satisfied in the intermediate-temperature range around $120 \mathrm{~K}$ [Fig. 8(a)]. Meanwhile, the predictions of Eq. (4) shown in Figs. 8(b) and 8(c) provide results consistent with Guyer's criterion in Eq. (3) that the hydrodynamic transport occur at low temperature in both PA-I and PA-II, which further validates our proposed criterion. Based on Eqs. (2) and (4), we further show in Figs. 8(d)-8(f) the transport diagram for three types of crystalline polymers. The hydrodynamic phonon transport in both PA-I and PA-II occurs in the low-temperature range below $50 \mathrm{~K}$, which is much higher than the observed temperature in other bulk materials, such as $\mathrm{NaF}$ [19] and liquid helium [20]. In PE, the hydrodynamic regime locates in the intermediate-temperature range from 50 to $300 \mathrm{~K}$. This unique hydrodynamic behavior in PE originates from the sudden change in umklapp and normal scattering rates in that temperature range [Fig. 8(a)].

\section{COLLECTIVE MOTION}

Finally, we discuss the collective drifting motion of phonons in crystalline polymers, which is another unique signature of hydrodynamic phonon transport as a result of the dominant momentum-conserved normal process $[8,16]$. Under a temperature gradient, phonons would equilibrate toward a displaced Bose-Einstein distribution $n_{d}=$ $1 /\left\{\exp \left[\frac{\hbar(\omega-\mathbf{q} \cdot \mathbf{u})}{k_{B} T}\right]-1\right\}$, where $\mathbf{q}$ denotes the phonon wave vector. The collective drift velocity $\mathbf{u}$ is constant for all phonon modes. Assuming a small temperature gradient and drift velocity, the phonon distribution function can be further linearized as [14]

$$
n_{d}=n_{0}+n_{0}\left(n_{0}+1\right) \frac{\hbar}{k_{B} T}(\mathbf{q} \cdot \mathbf{u}) .
$$

A normalized form is used in the following discussion as $\overline{d n}=\left(n_{d}-n_{0}\right) /\left[n_{0}\left(n_{0}+1\right)\right]$, where $n_{d}$ is calculated from the iterative solution of BTE [16]. Under the fully collective drifting condition, the normalized drifting term $\overline{d n}$ has a linear dependence on the wave vector along the transport direction $q_{z}$ as $\frac{\hbar q_{z} u}{k_{B} T}$. To quantify how close the calculated phonon distribution $n$ is to the ideal displaced distribution $n_{d}$, Ding et al. [16] defined a phonon drifting component as

$$
\rho_{d}=\frac{\sum C(\omega) \overline{d n} q_{z}}{\sqrt{\sum C(\omega) \overline{d n}^{2}} \sqrt{\sum C(\omega) q_{z}^{2}}} \times 100 \% .
$$



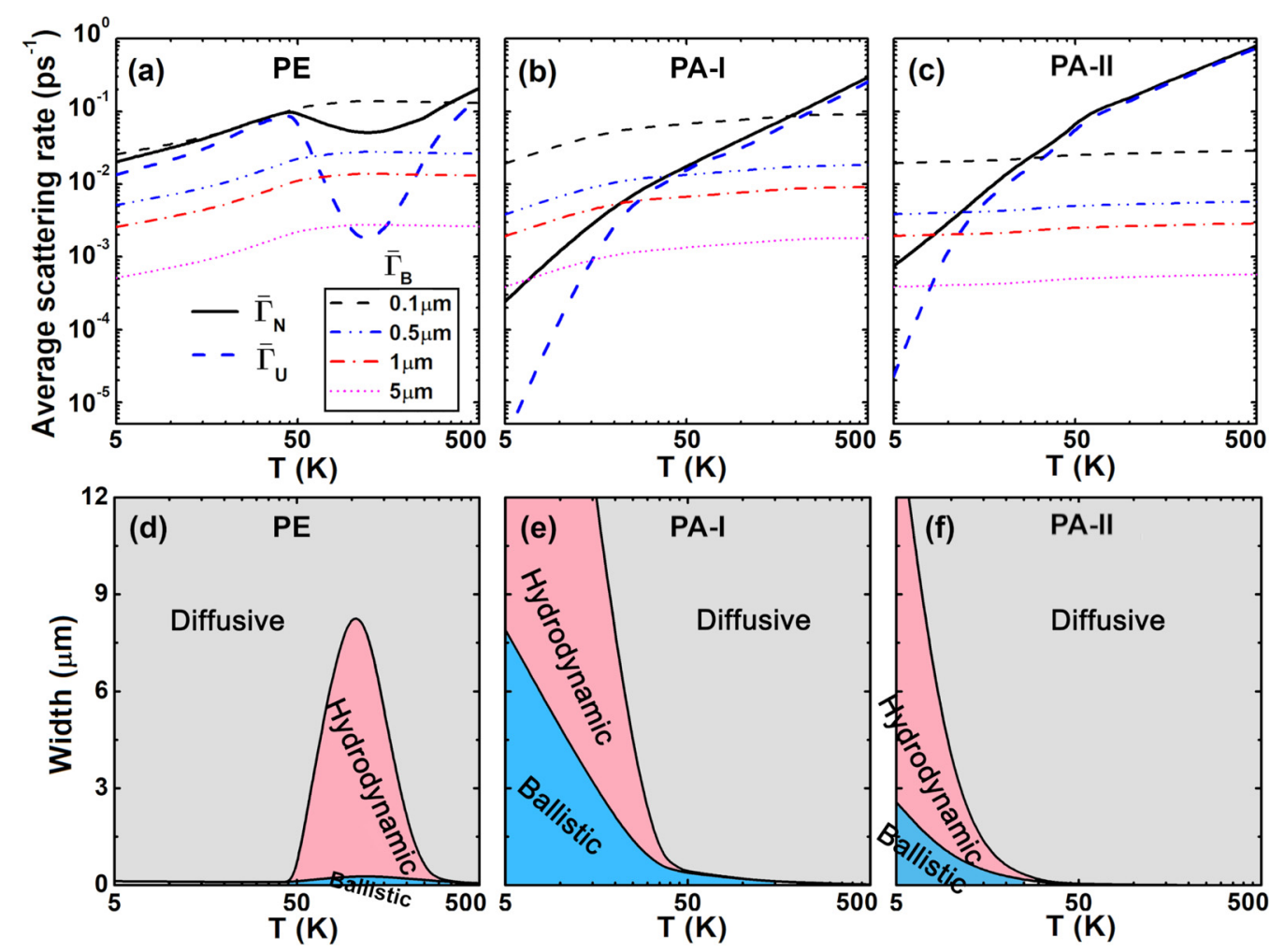

FIG. 8. The averaged scattering rates of the normal process, umklapp process, and boundary scattering calculated based on Eq. (4) for (a) PE, (b) PA-I, and (c) PA-II. Schematic diagrams of the ballistic, hydrodynamic, and diffusive regimes for (d) PE, (e) PA-I, and (f) PA-II.

Thus, for an ideally displaced distribution, the phonon drifting component $\rho_{d}$ should reach a value of $100 \%$. However, Fig. 3(c) shows that $\rho_{d}$ in PE calculated based on Eq. (6) is below $50 \%$ for the entire temperature range and fails to capture at all the hydrodynamic transport in the intermediatetemperature range, as shown in the phase diagram in Fig. 8(d). We believe that such a failure of Eq. (6) has the same cause as the failure of the thermodynamic average of Eq. (3) in PE. Therefore, we redefine $\rho_{d}$ based on the weighting function of $\kappa(\omega)$ as

$$
\rho_{d}=\frac{\sum \kappa(\omega) \overline{d n} q_{z}}{\sqrt{\sum \kappa(\omega) \overline{d n}^{2}} \sqrt{\sum \kappa(\omega) q_{z}^{2}}} \times 100 \% .
$$

With this new definition, Fig. 3(c) shows that $\rho_{d}$ calculated by Eq. (7) is closer to $100 \%$ at low temperatures for both PA-I and PA-II, while the trend predicted by Eq. (7) is consistent with the prediction by Eq. (6) for both PA-I and PA-II [Fig. 3(c)]. Especially for the case of PE, the sudden increase of $\rho_{d}$ above $50 \mathrm{~K}$ can well capture the onset of hydrodynamic transport, and the peak temperature for hydrodynamic transport predicted by Eq. (7) agrees quantitatively well with that shown in the phase diagram of Fig. 8(c).

\section{SUMMARY}

In this work, based on the Boltzmann transport equation, we have studied hydrodynamic phonon transport in organic materials, i.e., bulk crystalline polymers. In both PA-I and PA-II, notable hydrodynamic transport takes place in the low-temperature range up to $50 \mathrm{~K}$. Surprisingly, a weak hydrodynamic behavior emerges in $\mathrm{PE}$ at the intermediatetemperature range around $120 \mathrm{~K}$, an abnormal behavior that cannot be captured by Guyer's criterion for predicting hydrodynamic phonon transport. By analyzing the spectral phonon properties, we found that a flat band of the torsional motion exists in the phonon dispersion of PE due to its high structural anisotropy and cylinder structure. This flat band hybridizes strongly with acoustic phonons in PE, which lowers the frequency for acoustic phonons and enhances phonon-phonon scattering for low-frequency phonons, leading to suppressed thermal transport for low-frequency phonons. Consequently, the dominant phonon frequency for thermal conductivity shifts to a higher frequency and separates completely from the dominant peak frequency of the specific heat, causing the breakdown of Guyer's criterion in PE. A modified criterion based on the spectral thermal conductivity was proposed to determine the emergence of hydrodynamic transport, which was found to work excellently in all three kinds of polymer crystals, providing quantitative agreements with the predictions from both thermal conductivity results and the phonon drifting component. Our study provides a reliable criterion to predict the emergence of hydrodynamic phonon transport in complex systems, which will be helpful for the study of transport physics in new materials.

\section{ACKNOWLEDGMENTS}

This project is supported in part by grants from the National Natural Science Foundation of China (Grants No. 12075168 and No. 11890703), the Science and 
Technology Commission of Shanghai Municipality (Grants No. 19ZR1478600 and No. 18JC1410900), the Fundamental Research Funds for the Central Universities (Grant No. 22120200069), and the Open Fund of Hunan Provincial Key
Laboratory of Advanced Materials for New Energy Storage and Conversion (Grant No. 2018TP1037_201901). Z.Z. thanks the China Scholarship Council (CSC) for financial support.
[1] N. Li, J. Ren, L. Wang, G. Zhang, P. Hänggi, and B. Li, Rev. Mod. Phys. 84, 1045 (2012).

[2] X. Gu, Y. Wei, X. Yin, B. Li, and R. Yang, Rev. Mod. Phys. 90, 041002 (2018).

[3] Z. Zhang and J. Chen, Chin. Phys. B 27, 35101 (2018).

[4] Z. Zhang, Y. Ouyang, Y. Cheng, J. Chen, N. Li, and G. Zhang, Phys. Rep. 860, 1 (2020).

[5] X. Xu, L. F. Pereira, Y. Wang, J. Wu, K. Zhang, X. Zhao, S. Bae, C. Tinh Bui, R. Xie, J. T. Thong, B. H. Hong, K. P. Loh, D. Donadio, B. Li, and B. Ozyilmaz, Nat. Commun. 5, 3689 (2014).

[6] R. A. Guyer and J. A. Krumhansl, Phys. Rev. 148, 778 (1966).

[7] M. Markov, J. Sjakste, G. Barbarino, G. Fugallo, L. Paulatto, M. Lazzeri, F. Mauri, and N. Vast, Phys. Rev. Lett. 120, 075901 (2018).

[8] Y. Guo and M. Wang, Phys. Rep. 595, 1 (2015).

[9] V. Martelli, J. L. Jiménez, M. Continentino, E. BaggioSaitovitch, and K. Behnia, Phys. Rev. Lett. 120, 125901 (2018).

[10] V. Narayanamurti and R. C. Dynes, Phys. Rev. Lett. 28, 1461 (1972).

[11] N. N. Zholonko, Phys. Solid State 48, 1678 (2006).

[12] A. Cepellotti, G. Fugallo, L. Paulatto, M. Lazzeri, F. Mauri, and N. Marzari, Nat. Commun. 6, 6400 (2015).

[13] Y. Zhou, X. Zhang, and M. Hu, Nano Lett. 17, 1269 (2017).

[14] S. Lee, D. Broido, K. Esfarjani, and G. Chen, Nat. Commun. 6, 6290 (2015).

[15] S. Huberman, R. A. Duncan, K. Chen, B. Song, V. Chiloyan, Z. Ding, A. A. Maznev, G. Chen, and K. A. Nelson, Science 364, 375 (2019).

[16] Z. Ding, J. Zhou, B. Song, V. Chiloyan, M. Li, T. H. Liu, and G. Chen, Nano Lett. 18, 638 (2018).

[17] Y. Machida, N. Matsumoto, T. Isono, and K. Behnia, Science 367, 309 (2020).

[18] H. Beck, P. F. Meier, and A. Thellung, Phys. Status Solidi 24, 11 (1974).

[19] H. E. Jackson, C. T. Walker, and T. F. McNelly, Phys. Rev. Lett. 25, 26 (1970).

[20] C. C. Ackerman, B. Bertman, H. A. Fairbank, and R. A. Guyer, Phys. Rev. Lett. 16, 789 (1966).

[21] N. Shulumba, O. Hellman, and A. J. Minnich, Phys. Rev. Lett. 119, 185901 (2017).

[22] A. Henry and G. Chen, Phys. Rev. Lett. 101, 235502 (2008).
[23] X. Xu, J. Chen, J. Zhou, and B. Li, Adv. Mater. 30, 1705544 (2018).

[24] X. Xu, J. Zhou, and J. Chen, Adv. Funct. Mater. 30, 1904704 (2020).

[25] X. Wang, M. Kaviany, and B. Huang, Nanoscale 9, 18022 (2017).

[26] A. Jain, S. P. Ong, G. Hautier, W. Chen, W. D. Richards, S. Dacek, S. Cholia, D. Gunter, D. Skinner, G. Ceder, and K. A. Persson, APL Mater. 1, 011002 (2013).

[27] M. Omini and A. Sparavigna, Phys. B (Amsterdam, Neth.) 212, 101 (1995).

[28] A. Ward, D. A. Broido, D. A. Stewart, and G. Deinzer, Phys. Rev. B 80, 125203 (2009).

[29] W. Li, J. Carrete, N. A. Katcho, and N. Mingo, Comput. Phys. Commun. 185, 1747 (2014).

[30] L. Lindsay and D. A. Broido, J. Phys. Condens. Matter 20, 165209 (2008).

[31] S. J. Stuart, A. B. Tutein, and J. A. Harrison, J. Chem. Phys. 112, 6472 (2000).

[32] B. Ni, T. Watanabe, and S. R. Phillpot, J. Phys. Condens. Matter 21, 084219 (2009).

[33] S. Shen, A. Henry, J. Tong, R. Zheng, and G. Chen, Nat. Nanotechnol. 5, 251 (2010).

[34] Y. Ouyang, Z. Zhang, Q. Xi, P. Jiang, W. Ren, N. Li, J. Zhou, and J. Chen, RSC Adv. 9, 33549 (2019).

[35] L. Lindsay, D. A. Broido, and N. Mingo, Phys. Rev. B 80, 125407 (2009).

[36] A. Cepellotti and N. Marzari, Phys. Rev. X 6, 041013 (2016).

[37] L. Lindsay and D. A. Broido, Phys. Rev. B 84, 155421 (2011).

[38] M. Kazan, G. Guisbiers, S. Pereira, M. R. Correia, P. Masri, A. Bruyant, S. Volz, and P. Royer, J. Appl. Phys. 107, 083503 (2010).

[39] J. Callaway, Phys. Rev. 113, 1046 (1959).

[40] H. Casimir, Physica 5, 495 (1938).

[41] L. Lindsay, D. A. Broido, and N. Mingo, Phys. Rev. B 82, 115427 (2010).

[42] B. Szigeti and H. Fröhlich, Proc. R. Soc. London, Ser. A 264, 198 (1961).

[43] K. H. Matlack, A. Bauhofer, S. Krödel, A. Palermo, and C. Daraio, Proc. Natl. Acad. Sci. USA 113, 8386 (2016).

[44] Z. Zhang, J. Chen, and B. Li, Nanoscale 9, 14208 (2017).

[45] S. Xiong, K. Saaskilahti, Y. A. Kosevich, H. Han, D. Donadio, and S. Volz, Phys. Rev. Lett. 117, 025503 (2016). 